\title{
Uterus didelphys with pregnancy and its different maternal and perinatal outcomes
}

\author{
Rajshree Dayanand Katke ${ }^{1 *}$, Sivanandini Acharya ${ }^{2}$, Soni Mourya ${ }^{2}$
}

\begin{abstract}
${ }^{1}$ Department of Obstetrics and Gynecology, Suprintendent Cama and Albless Hospital, Mumbai, Maharashtra, India ${ }^{2}$ Department of Obstetrics and Gynecology, J. J. Group of Hospital, Mumbai, Maharashtra, India
\end{abstract}

Received: 15 April 2017

Revised: 20 August 2017

Accepted: 23 August 2017

\section{*Correspondence:}

Dr. Rajshree Dayanand Katke,

E-mail: drrajshrikatke@gmail.com

Copyright: (c) the author(s), publisher and licensee Medip Academy. This is an open-access article distributed under the terms of the Creative Commons Attribution Non-Commercial License, which permits unrestricted non-commercial use, distribution, and reproduction in any medium, provided the original work is properly cited.

\begin{abstract}
Mullerian duct anomalies (MDAs) are congenital defects of the female genital system that arise from abnormal embryological development of the Mullerian ducts. A didelphys uterus, also known as a "double uterus," is one of the least common amongst MDAs. These abnormalities can include failure of development, fusion, canalization, or reabsorption, which normally occurs between 6 and 22 weeks in utero. Most sources estimate an incidence of these abnormalities to be from 0.5 to $5.0 \%$ in the general population. It is an observational study of cases of uterine didelphys with pregnancy over a period of 2 years. Uterus didelphys with pregnancy has variable maternal and perinatal outcome. First case presents an uneventful course in pregnancy which was terminated with caesarian section with a healthy baby. Second case presented to us with retained placenta with perforation of left horn which was managed by obstetric hysterectomy. The third case presents a didelphys uterus with a congenitally abnormal fetus with Hydrops fetalis with IUFD at $7^{\text {th }}$ month of gestation terminated by caesarian section due to associated vertical vaginal septum. Pregnancy in a uterus Didelphys deserves early diagnosis of the anomaly, and meticulous care in pregnancy and delivery to avert the associated adverse outcomes.
\end{abstract}

Keywords: Caesarian section, Mullerian duct, Obstetric hysterectomy, Perforation, Uterus didelphys, Vertical vaginal septum

\section{INTRODUCTION}

Mullerian duct anomalies can include failure of development, fusion, or reabsorption, which normally occurs between 6 and 22 weeks in utero. Most sources estimate an incidence of these abnormalities to be from 0.5 to $5.0 \%$ in the general population.

The most recent and widely used classification systems for the different types of Mullerian duct abnormalities were created by Buttram Jr. and Gibbons (1979) and the American Fertility Society (1988). A didelphys uterus is characterized by complete failure of the Mullerian ducts to fuse leading to separate uterine cavities and two cervices. A longitudinal vaginal septum is also present that may range from thin and easily displaced to thick and inelastic.

Initial suspicion of the condition followed by the diagnosis usually begins with a routine speculum exam where visualization of anatomical abnormalities warrants further investigation.

Further, because the Mullerian ducts develop often in association with Wolffian ducts, abnormalities of the kidneys may be found in conjunction with uterine abnormalities. 


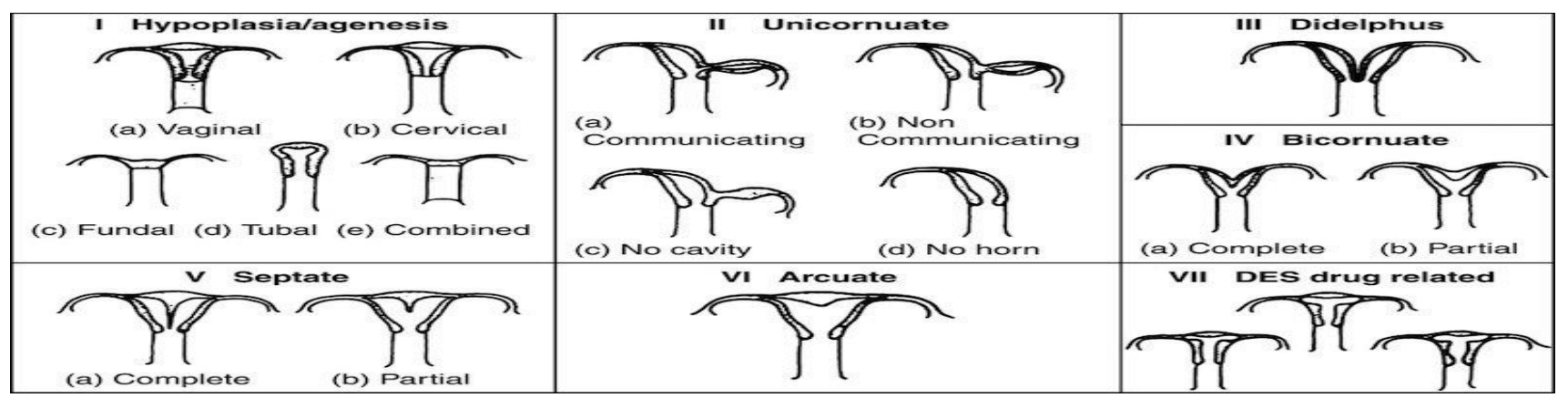

Figure 1: American fertility society classification of uterine anomalies.

\section{CASE REPORT}

\section{Case 1}

The patient was a case of 32 years old female, gravida 2 abortion 1 who came to our antenatal OPD as a known case of didelphys uterus with infertility conception at 2 nd month of gestation. Patient was diagnosed as a case of didelphys uterus on sonography after first spontaneous abortion. At the first visit, her general examination was within normal limit. Per abdominally, uterus was not palpable, there was no tenderness, guarding or rigidity. Per speculum examination revealed a vertical vaginal septum and two cervices were visualized on either side of the septum. Per vaginal examination confirmed the presence of two cervix. An obstetric ultrasound was done which revealed two distinct widely separated uterine bodies with a single viable gestation of 8.6 weeks in right side. Patient was adviced the routine antenatal investigations which were within normal limit and was adviced bed rest and progesterone support. Patient was followed with routine antenatal checkup and obstetric USG for fetal well-being.

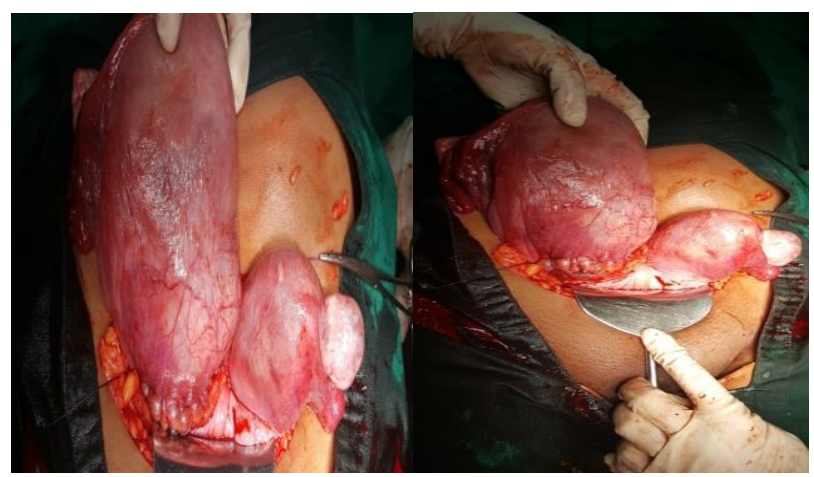

Figure 2: Uterus didelphys with pregnancy in right side.

The antenatal course was uneventful. USG at 37 weeks revealed a full-term pregnancy with estimated fetal weight of $2.8 \mathrm{~kg}$ with cephalic presentation. Patient was taken for elective LSCS in view of precious pregnancy at 37 weeks. Intraoperative finding was of two separate uterus with two separate fallopian tubes and ovary with pregnancy on the right side (Figure 2).

Baby was delivered by lower segment caesarian section, female baby of $2.6 \mathrm{~kg}$ weight. Post-operative period was uneventful. Both mother and baby were healthy at discharge.

\section{Case 2}

A 28-year-old female P3L3 day 12 of full term normal delivery was referred to our hospital ivo retained placenta. Patient was complaining of bleeding $\mathrm{p} / \mathrm{v}$ and pain in abdomen since the day of delivery. Patient gave the history of full term vaginal delivery at home 12 days back with no expulsion of placenta postdelivery. Patient went to some local practitioner where $\mathrm{d}$ and $\mathrm{c}$ was attempted 2 days back after which she was referred to our centre.

On general examination patient was febrile with pulse of 110/min and BP of 90/60mmhg. P/A examination showed uterus of 18 -week size with mild tenderness. On $\mathrm{p} / \mathrm{s}$ examination reddish brown discharge seen and $\mathrm{p} / \mathrm{v}$ exam showed uterus of 18 -week size with cervical motion tenderness. Ultrasound examination showed two endometrial canals with $10 \times 4 \times 5 \mathrm{~cm}$ echogenic lesion present in left uterine cavity with thinning of uterine myometrium along left posterolateral wall suspected retained placenta with sealed rupture near left fundus with organised blood in POD. 


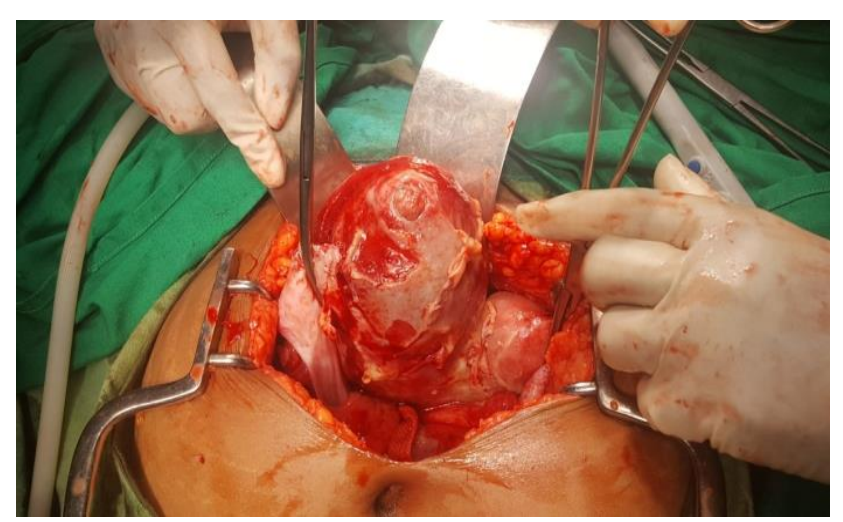

Figure 3: Perforation in left fundal area with pus flakes.

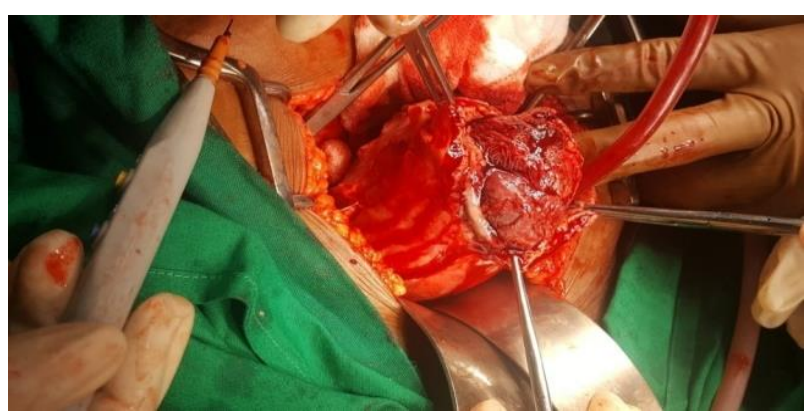

Figure 4: Placenta visualized from site of perforation.

Patient underwent exploratory laparotomy with obstetric hysterectomy. In situ findings showed didelphys uterus with retained placenta in left uterine cornua with uterine perforation, and pus discharge from perforation site (Figure 3, 4, 5).

Bowel and omentum adherent to the left fundal area. Obstetric hysterectomy with peritoneal wash given. Postoperative period was covered by higher antibiotics. Patient recovered well and was discharged healthy.

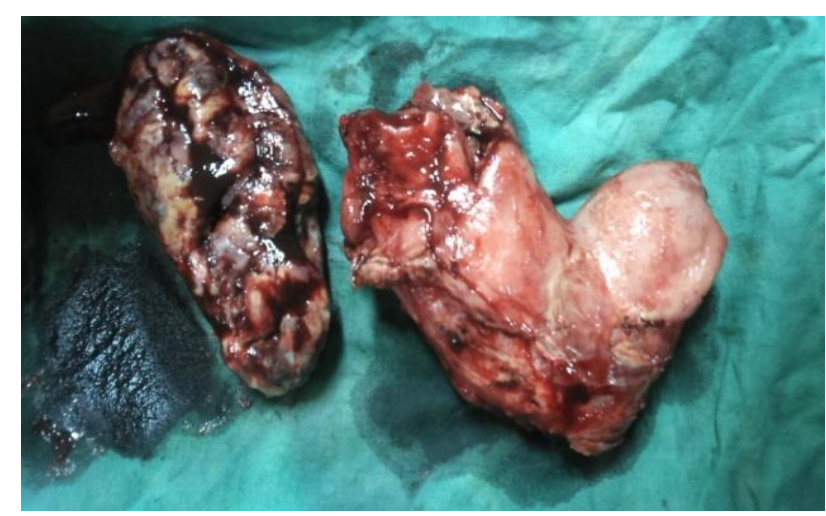

Figure 5: Post obstetric hysterectomy specimen of uterus didelphys with placenta.

\section{Case 3}

A 30-year-old G2P1L1 with 26 weeks gestation came referred to our hospital ivo intrauterine fetal demise of 23.3 weeks with features s/o of Hydrops fetalis. Patient gave history of spotting $\mathrm{p} / \mathrm{v}$ since one-day USG showed IUFD of 23.3 weeks with hydrops fetalis.

Patient had 1 living issue of 7 years by LSCS done ivo? uterus didelphys. On general examination patient was vitally stable, per abdominal examination revealed uterus of 32-week size with no FHS localised, in a relaxed state. $\mathrm{P} / \mathrm{S}$ examination revealed a vertical vaginal septum with two cervices on either side with minimal spotting on right side. $\mathrm{P} / \mathrm{V}$ examination confirmed the findings.

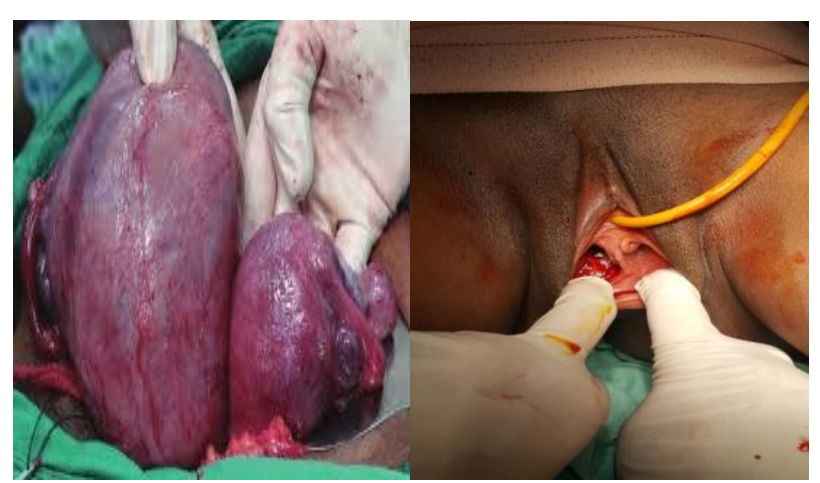

Figure 6: Didelphys uterus with vertical vaginal septum.

All routine investigations were within normal limits. Repeat ultrasound examination showed uterus diadelphys with right sided gestation with IUFD of 24 wks with hydrops fetalis. In situ findings during LSCS confirmed the diagnosis of uterus diadelphys showing a gravid right sided uterus (Figure 6). Baby delivered by lscs. Postoperative period was uneventful.

\section{DISCUSSION}

Uterine anomalies are associated with an increase in cases of breech presentation, premature labor, abnormal presentation with dystocia, and the necessity for cesarean section. ${ }^{1}$ Although pregnancy period may be uneventful, it is possible that uterine anomalies produce a considerable lower percentage of viable babies. The spontaneous abortion rates are estimated at $43 \%$, the premature birth rate is approximately $38 \%$ and the fetal survival rate is approximately $50 \%$ patients with uterus didelphys, thus it belongs to a high-risk group and deserve a particular prenatal care. ${ }^{2}$

Therefore, it is of great importance for the clinical management of these cases that abnormalities of the reproductive tract are detected in early stage.

The modalities for correct diagnosis frequently used include invasive methods such as hysteroscopy, hysterosalpingography, and laparoscopy/laparotomy. Diagnosis of bicornuate uterus made on 
hysterosalpingogram by radiologists is often incorrect and needs to be revised. ${ }^{3}$ A $2 \mathrm{D}$ ultrasound is usually the first type of imaging done. The use of 3D ultrasound is becoming more commonly used for diagnosis as it overcomes the limitation of 2D ultrasound. Magnetic resonance imaging is accurate and valuable as it is noninvasive and can diagnose associated urinary tract abnormalities at the same time. The septate uterus is most common at $35 \%$ followed by bicornuate at $25 \%$, then arcuate at $20 \%$, then unicornuate at $9.6 \%$, and complete agenesis at $3 \% .{ }^{4}$ Heinonen PK, evaluated the long-term clinical consequences, and reproductive performances of 49 women with uterus Didelphys that were followed up to 6.3 years. $^{1}$ According to the study the recurrent miscarriage rate is $21 \%$, ectopic pregnancy rate is $2 \%$, fetal survival rate is $75 \%$, prematurity is $24 \%$, IUGR $11 \%$, caesarian rate is $84 \% .^{5}$

\section{CONCLUSION}

Pregnancy in a uterus Didelphys deserves early diagnosis of the anomaly, and meticulous care in pregnancy and delivery to avert the associated adverse outcomes. Clinicians should have high index of suspicion of uterine anomaly to make early diagnosis of uterus Didelphys especially while handling obstetric complications.

Funding: No funding sources Conflict of interest: None declared

\section{Ethical approval: Not required}

\section{REFERENCES}

1. Green LK, Harris RE. Uterine anomalies; frequency of diagnosis, and obstetric complications. 1976;47 (4):427-8.

2. Grimbizis GF, Camus M, Tarlatzis BC, Bontis JN, and Devroey P. Clinical implications of uterine malformations and hysteroscopic treatment results. Human Reproduction Update. 2001;7(2)161-74.

3. Sheth SS, Sonkawde R. Uterine septum misdiagnosed on hysterosalpingogram. Int J Gynecol Obstet. 2000;69(3):261-3.

4. Ng PC, Kann KS. Cervical ectopic pregnancy in a 23 year old with uterus didelphys. Clin Pract Cases Emergency Med. 2017

5. Heinonen PK. Clinical implications of the didelphic uterus: long-term follow-up of 49 cases. Eur J Obstet Gynecol Reprod Biol. 2000;91(2)183-90.

Cite this article as: Katke RD, Acharya S, Mourya S. Uterus didelphys with pregnancy and its different maternal and perinatal outcomes. Int J Reprod Contracept Obstet Gynecol 2017;6:4690-3. 